\title{
Parasitismo de traça-das-crucíferas por Oomyzus sokolowskii
}

\author{
Christian Sherley Araújo da Silva-Torres ${ }^{(1)}$, Jorge Braz Torres ${ }^{(1)}$, Reginaldo Barros ${ }^{(1)}$ e Ângelo Pallini ${ }^{(2)}$ \\ (1) Universidade Federal Rural de Pernambuco, Departamento de Agronomia, Av. Dom Manoel de Medeiros, s/no, Dois Irmãos, \\ CEP 52171-900 Recife, PE. E-mail: sherleyjbt@yahoo.com, jtorres@depa.ufrpe.br, rbarros@depa.ufrpe.br (2)Universidade Federal de \\ Viçosa, Departamento de Biologia Animal, Av. P. H. Rolfs, s/no, CEP 36570-000 Viçosa, MG. E-mail: pallini@ufv.br
}

Resumo - O objetivo deste trabalho foi avaliar a capacidade de parasitismo do parasitoide larval-pupal Oomyzus sokolowskii, submetido a diferentes densidades do hospedeiro traça-das-crucíferas (Plutella xylostella), em laboratório, casa telada e campo. Em laboratório e campo, O. sokolowskii foi exposto às densidades de 2, 4, 8 e 16 lagartas. Em laboratório, empregaram-se recipientes de $300 \mathrm{~mL}$ e, em campo, plantas de couve foram confinadas em gaiolas de organza $(30 \times 50 \mathrm{~cm})$. Em casa telada, foram utilizadas microparcelas compostas por seis plantas de repolho infestadas com 25, 50, 85 ou 100 lagartas. O número de lagartas parasitadas aumentou de acordo com a densidade do hospedeiro e variou de 1,7 a 10,4, em laboratório, e de 0,61 a 7,0, em campo. Em casa telada, a maior taxa de parasitismo foi observada nas microparcelas com densidades mais elevadas do hospedeiro. O tempo de exposição aos parasitoides proporcionou maior taxa de parasitismo após 72 horas (24 horas, 52,4\% e 72 horas, 80,7\%) independentemente da densidade. Oomyzus sokolowskii responde positivamente ao incremento na densidade de . xylostella, embora a taxa de parasitismo permaneça constante independentemente da disponibilidade do hospedeiro.

Termos para indexação: Plutella xylostella, controle biológico, densidade do hospedeiro, reposta funcional.

\section{Parasitism of diamondback moth by Oomyzus sokolowskii}

\begin{abstract}
The objective of this work was to evaluate the parasitism capacity of the larval-pupal parasitoid Oomyzus sokolowskii submitted to different densities of the host diamondback moth, Plutella xylostella, in the laboratory, greenhouse and field. In the laboratory and in the field, O. sokolowskii was exposed to densities of 2, 4, 8 or 16 larvae. In the laboratory, $300 \mathrm{~mL}$ recipient were used, and in the field kale plants were placed in cages $(30 \times 50 \mathrm{~cm})$. In the greenhouse, microparcels consisted of six cabbage plants each infested with 25 , 50,85 or 100 larvae. The number of parasitized larvae increased with host density and varied from 1.7 to 10.4 (laboratory) and from 0.61 to 7.0 (field). In the greenhouse, parasitism rate was higher in microparcels with higher host infestation. The time of exposition was significant, with higher parasitism rate at 72 hours after host exposure (24 hour, 52.4\%; and 72 hour, 80.7\%), irrespective of host density. Oomyzus sokolowskii responds to increases in host density, but maintains the parasitism rate unchanged irrespective of variations in the host availability.
\end{abstract}

Index terms: Plutella xylostella, biological control, host density, functional response.

\section{Introdução}

A resposta de parasitoides relacionada à densidade do hospedeiro pode ser funcional e numérica. A resposta funcional relaciona a densidade do hospedeiro com o número de hospedeiros parasitados pelo parasitoide, ao passo que a resposta numérica relaciona o número de indivíduos do parasitoide, pela agregação e maior multiplicação, com o aumento da densidade do hospedeiro (Holling, 1961). Consequentemente, ambas as respostas são fundamentais na dinâmica populacional hospedeiro-parasitoide, pois podem indicar a capacidade de um parasitoide de regular a densidade do hospedeiro (Schenk \& Bacher, 2002).

A modelagem de dados que emprega a resposta funcional sugere que parasitoides podem ser atraídos e exibir comportamento de agregação em locais de densidade mais elevada do hospedeiro (Hassell, 1978). No entanto, parasitoides também devem ser capazes de localizar hospedeiros em ambientes com baixa densidade (Waage, 1983), uma vez que essa capacidade proporciona sincronia entre o crescimento populacional do parasitoide e da praga. Assim, uma vez localizados, os hospedeiros podem ser explorados

Pesq. agropec. bras., Brasília, v.45, n.7, p.638-645, jul. 2010 
por parasitoides em diferentes proporções, em função da sua densidade, tempo de exposição e distribuição no ambiente (Foerster et al., 2001; Pratissoli et al., 2005). Consequentemente, conhecer como os parasitoides respondem às variações da distribuição e da densidade dos hospedeiros é essencial para que se determine a sua eficiência como agente de controle biológico.

O endoparasitoide gregário Oomyzus sokolowskii (Kurdjumov) (Hymenoptera: Eulophidae) tem sido citado por exercer controle natural da traça-das-crucíferas, Plutella xylostella (Linnaeus) (Lepidoptera: Plutellidae) em várias regiões produtoras de brássicas do mundo (Wang et al., 1999), inclusive no Agreste de Pernambuco (Ferreira et al., 2003; Silva-Torres et al., 2009a). No entanto, pouco se conhece sobre o seu potencial de controle. Portanto, o conhecimento sobre a capacidade de resposta (parasitismo) de $O$. sokolowskii a variações na densidade do hospedeiro poderá fornecer dados acerca de parâmetros comportamentais envolvidos neste processo, possibilitando a simulação de estimativas que poderão indicar o real potencial de sucesso deste parasitoide em colonizar habitats infestados pela praga.

Espera-se que fêmeas de O. sokolowskii sejam capazes de responder prontamente ao incremento na densidade de $P$. xylostella, parasitando proporcionalmente mais lagartas quanto maior for a disponibilidade no ambiente. Adicionalmente, esperase que $O$. sokolowskii aumente sua população por meio de seus descendentes e, com isso, tenha potencial de reduzir efetivamente a população dessa praga antes de causar prejuízo econômico.

O objetivo deste trabalho foi avaliar a capacidade de parasitismo do parasitoide larval-pupal O. sokolowskii, quando submetido a diferentes densidades do seu hospedeiro, a traça-das-crucíferas ( $P$. xylostella), em laboratório, casa telada e campo.

\section{Material e Métodos}

Os insetos utilizados nos experimentos foram obtidos de colônias mantidas no Laboratório de Ecologia de Insetos e Controle Biológico da Universidade Federal Rural de Pernambuco (UFRPE). Fêmeas do parasitoide utilizadas nos experimentos foram previamente acasaladas e alimentadas, no estágio de 48-72 horas de idade, e, dependendo do experimento, encontravam-se entre $35^{\mathrm{a}}$ e a $40^{\mathrm{a}}$ geração em laboratório.

Para avaliar o efeito da densidade do hospedeiro no parasitismo de $O$. sokolowskii, em laboratório, foi instalado experimento em setembro de 2008. Utilizouse o delineamento inteiramente casualizado composto de quatro tratamentos, representados pelas densidades de 2, 4, 8 e 16 lagartas com 7, 7, 6 e 5 repetições, respectivamente. Cada repetição consistiu de um disco de folha de repolho (Brassica oleracea var. capitata) de $8 \mathrm{~cm}$ de diâmetro, acondicionado em recipiente de plástico de $300 \mathrm{~mL}$, sobre disco de papel-filtro de igual tamanho. Em seguida, lagartas de terceiro instar de P. xylostella foram liberadas nas respectivas densidades estudadas. Os recipientes foram fechados com tampa contendo uma abertura circular fechada com tecido telado, permitindo desse modo a circulação de ar. As condições de laboratório foram de $26 \pm 1^{\circ} \mathrm{C}$ de temperatura do ar, $70 \pm 10 \%$ de umidade relativa do ar e fotoperíodo de 13:11 horas (luz:escuro).

Uma hora após a infestação dos discos de folhas com as lagartas, dez fêmeas do parasitoide foram liberadas no interior de cada recipiente e permaneceram por 48 horas, procedendo-se, em seguida, à individualização das lagartas em tubos de vidro contendo fragmento de folha de repolho. Os vidros foram fechados com filme de plástico de PVC transparente ALPFILM (ALPES Indústria e Comércio de Plásticos LTDA., São Paulo, Brasil) e mantidos nesse recipiente por um período de dez dias. Avaliou-se a emergência de adultos de $P$. xylostella ou do parasitoide, sendo quantificado o número de lagartas parasitadas e o número de descendentes produzidos por lagarta em função das densidades de 2 a 16 lagartas oferecidas ao parasitoide. As pupas que não originaram parasitoides ou mariposas foram abertas para determinação da ocorrência ou não do parasitismo.

O número de lagartas parasitadas e a taxa de parasitismo foram comparados com relação às densidades de lagartas oferecidas por meio de análise de regressão (SAS Institute, 2000), utilizando-se o modelo que melhor representou os resultados mediante o coeficiente de determinação e a significância dos seus parâmetros.

O experimento em casa telada, para avaliar o efeito da densidade do hospedeiro no parasitismo de O. sokolowskii, foi conduzido com plantas cultivadas 
em microparcelas instaladas em dois telados da UFRPE, cada um medindo $6 \times 2 \times 2 \mathrm{~m}$ (comprimento $\mathrm{x}$ largura $\mathrm{x}$ altura), fechados lateralmente com tela antiafídica e cobertos com filme agrícola utilizado em casa de vegetação. As condições do ambiente, durante o período experimental, foram monitoradas com DataLogger WatchDog (Spectrum Technologies, Inc., Plainfield, Illinois, EUA) em intervalos de 30 minutos, registrando-se os seguintes valores: temperatura média do ar de $30 \pm 8,8^{\circ} \mathrm{C}$; temperatura mínima de $21,3^{\circ} \mathrm{C}$; temperatura máxima de $53,5^{\circ} \mathrm{C} ; 66,4 \pm 24,8 \%$ de umidade relativa do ar, e fotofase natural de, aproximadamente, 12 horas.

Cada telado possuia, de forma equidistante, quatro microparcelas, medindo $1,0 \mathrm{~m}$ de diâmetro por $0,5 \mathrm{~m}$ de altura. Em cada microparcela foram cultivadas seis plantas de repolho. Assim, cada telado continha um total de 24 plantas de repolho por repetição. Após o início da formação da cabeça das plantas de repolho, elas foram infestadas com lagartas de P. xylostella de terceiro instar. $\mathrm{O}$ experimento foi conduzido em delineamento inteiramente casualisado em parcela subdividida com múltipla escolha. Cada microparcela representava uma subparcela e recebeu aleatoriamente uma das quatro densidades de lagartas: 25, 50, 85 ou 100 lagartas de P. xylostella. Assim, cada telado representava uma parcela experimental contendo quatro densidades do hospedeiro como subparcelas, sendo uma densidade por microparcela.

A infestação das lagartas sobre as plantas foi feita no final da tarde e a liberação dos parasitoides ao amanhecer do dia seguinte. No dia da infestação das plantas com as lagartas, tubos contendo parasitoides foram levados para os telados para aclimatação. Os tubos contendo os parasitoides foram colocados dentro do telado sobre um suporte de madeira a aproximadamente $30 \mathrm{~cm}$ acima da altura das plantas e localizado entre as quatro microparcelas de forma equidistante. $\mathrm{O}$ total de 400 fêmeas do parasitoide foi liberado por telado, sendo cada liberação considerada como uma repetição. O tempo de exposição das lagartas aos parasitoides foi de 24 e 72 horas, com cinco e seis repetições, respectivamente, repetidas no tempo, durante o período de setembro a novembro de 2008. Após os respectivos tempos de exposição ao parasitismo, todas as lagartas foram coletadas das plantas, agora consideradas como lagartas recuperadas das microparcelas, e levadas ao laboratório, alimentadas diariamente com repolho e monitoradas até a emergência de adultos de $P$. xylostella ou de $O$. sokolowskii. Todas as pupas inviáveis, sem emergência do parasitoide ou mariposa, foram abertas para confirmação da ocorrência ou não do parasitismo.

O número de lagartas recuperadas por microparcela foi inferior ao número liberado em todas as densidades $(25,50,85$ e 100). Portanto, a taxa de parasitismo igual a 100 (lagartas parasitadas/lagartas recuperadas) foi utilizada para avaliar o efeito da densidade no parasitismo. Os dados referentes à taxa de parasitismo foram submetidos a testes de normalidade (Kolmogorov D: normal test) e homogeneidade de variância (Bartlett's test), após os quais foram transformados em arco seno $(\mathrm{x} / 100)^{0,5}$, para atender aos pré-requisitos da análise de variância (SAS Institute, 2000).

As taxas de parasitismo, decorridas 24 e 72 horas da liberação do parasitoide, em função das densidades do hospedeiro, foram comparadas pelo teste $t$, utilizandose o PROC TEST do SAS. Além disso, as taxas de parasitismo em função da densidade de lagartas recuperadas, bem como o número de descendentes produzidos às 24 e 72 horas, após a liberação dos parasitoides, foram submetidos à análise de regressão (SAS Institute, 2000), utilizando-se o modelo que melhor se ajustou aos dados, mediante coeficiente de determinação e significância dos parâmetros. Para inferir sobre o efeito do tempo de exposição (24 e 72 horas) na taxa de parasitismo em função da densidade de lagartas, as inclinações dos modelos ajustados foram testadas para igualdade empregandose o PROC MIXED do SAS (SAS Institute, 2000).

O experimento para avaliar o efeito da densidade do hospedeiro no parasitismo de $O$. sokolowskii em campo foi conduzido em plantio orgânico de couve-manteiga (B. oleracea var. acephala), de aproximadamente 0,75 ha, localizado no Município de Chã Grande, PE $\left(08^{\circ} 15^{\prime} 14^{\prime \prime} \mathrm{S}, 35^{\circ} 30^{\prime} 0^{\prime \prime} \mathrm{W}\right.$ e altitude de $\left.505 \mathrm{~m}\right)$, durante o mês de março de 2009. As condições de campo foram monitoradas com DataLogger WatchDog (Spectrum Technologies, Inc., Plainfield, Illinois, EUA) a intervalos de $30 \mathrm{~min}$, registrando-se os seguintes vaolres: temperatura média do ar de $25,3 \pm 4,0^{\circ} \mathrm{C}$; temperatura mínima de $19,8^{\circ} \mathrm{C}$; temperatura máxima de $35,3^{\circ} \mathrm{C}$; umidade relativa do ar de $79 \pm 16,7 \%$ e fotoperíodo natural de 12,5:11,5 horas (luz:escuro). 
Plantas de couve com aproximadamente $50 \mathrm{~cm}$ de altura foram selecionadas aleatoriamente na área do plantio, inspecionadas para a retirada de artrópodes e, posteriormente, confinadas em gaiolas cilíndricas de tecido organza $(30 \times 50 \mathrm{~cm})$. Utilizou-se o delineamento experimental de inteiramente casualizado, sendo cada densidade de lagarta um tratamento, e cada gaiola contendo as lagartas e os parasitoides uma unidade experimental. As plantas foram infestadas com lagartas do terceiro instar de P. xylostella nas densidades de 2, 4, 8 e 16 lagartas por planta, com 21, 17, 17 e 10 repetições, respectivamente. Em seguida, dez fêmeas do parasitoide $O$. sokolowskii foram liberadas no interior de cada gaiola, permanecendo por 72 horas. Após esse período, as lagartas foram coletadas, trazidas ao laboratório e monitoradas até a emergência de adultos de $P$. xylostella ou de $O$. sokolowskii. As pupas inviáveis, sem emergência do parasitoide ou mariposa, foram abertas para determinação da ocorrência ou não do parasitismo, e avaliou-se o número de lagartas parasitadas, bem como a produção de descendentes por hospedeiro parasitado.

O número de lagartas parasitadas, a taxa de parasitismo e o número de descendentes produzidos foram determinados por meio de análise de regressão (SAS Institute, 2000), selecionando-se o modelo que melhor se ajustou aos resultados mediante os valores do coeficiente de determinação e significância dos parâmetros.

\section{Resultados e Discussão}

O número de lagartas de $P$. xylostella parasitadas por O. sokolowskii durante 48 e 72 horas, em laboratório e campo, respectivamente, aumentou significativamente em função da densidade de lagartas ofertadas $(p<0,01)$ (Figura 1). A média de lagartas parasitadas variou de $1,71 \pm 0,18$ a $10,4 \pm 1,32$ no laboratório, enquanto, em campo, variou de $0,6 \pm 0,14$ a $7,0 \pm 0,63$, quando 2 a 16 lagartas foram expostas ao parasitoide. A taxa de parasitismo de lagartas, entretanto, não diferiu em função do aumento da densidade de lagartas, com média de 65 a $85 \%$ de parasitismo em laboratório e 30 a $40 \%$ em campo.

Em casa telada, o parasitismo de P. xylostella por $O$. sokolowskii foi influenciado pelas variações na densidade do hospedeiro e no tempo de exposição. De modo geral, foi observado maior número de lagartas parasitadas em função do número de lagartas recuperadas nas microparcelas em 24 e 72 horas $(\mathrm{p}<0,01)$ (Figura 2). O aumento na taxa de parasitismo foi superior quando as lagartas permaneceram expostas ao parasitismo por 72 horas $(\mathrm{p}<0,01)$.

As percentagens de parasitismo em função da densidade de lagartas recuperadas foram independentes

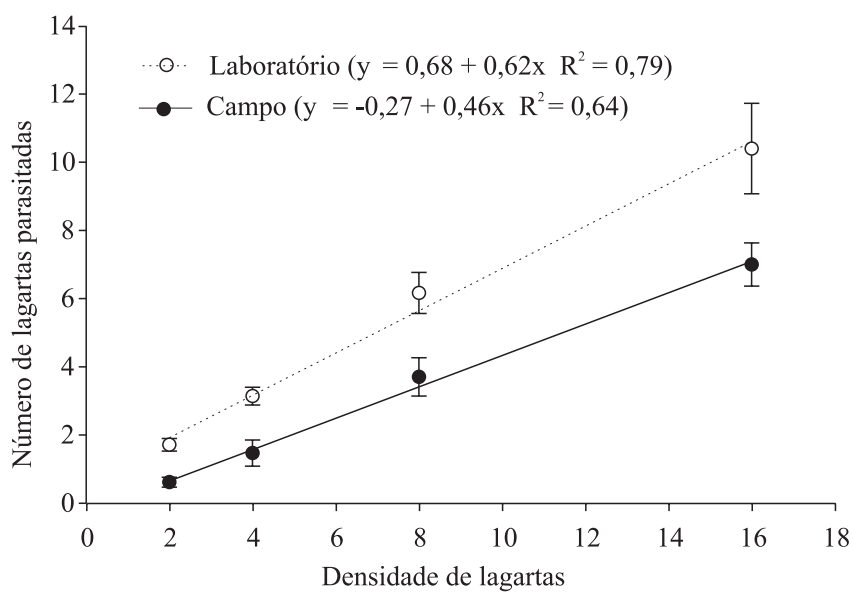

Figura 1. Número médio de lagartas de Plutella xylostella parasitadas durante 48 e 72 horas por Oomyzus sokolowskii, em função das diferentes densidades de lagartas de P. xylostella, em laboratório e campo, respectivamente. A barra vertical indica o erropadrão.

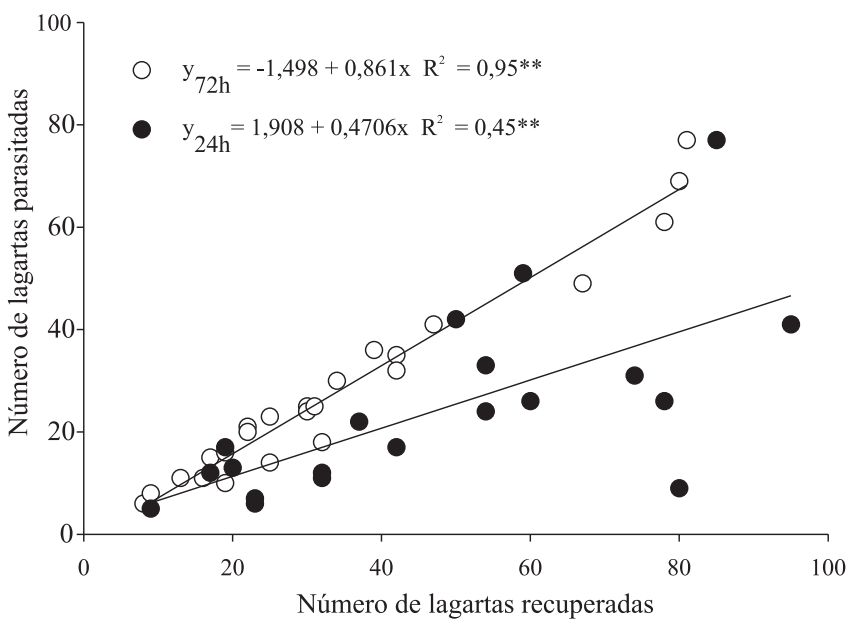

Figura 2. Número médio de lagartas de Plutella xylostella parasitadas por Oomyzus sokolowskii expostas por 24 ou 72 horas, em função do número de lagartas recuperadas de seis plantas por microparcela, em casa telada. **Significativo a $1 \%$ de probabilidade. 
da densidade inicial de lagartas por microparcela, sendo de $52,4 \pm 5,09 \%$ após 24 horas e de $80,7 \pm 2,46 \%$ após 72 horas $(\mathrm{p}<0,01)$. Considerando-se cada densidade de hospedeiro disponível separadamente $(25,50,85$ e 100), houve incremento significativo na taxa de parasitismo em função do tempo apenas nas densidades de 50 e 85 lagartas por microparcelas (Tabela 1). Nas densidades de 25 e 100 lagartas, houve incremento de aproximadamente $30 \%$ na taxa de parasitismo, no entanto essa diferença não foi significativa.

$\mathrm{O}$ aumento da taxa de parasitismo em função da densidade de hospedeiros disponíveis não foi observado em casa telada, de forma semelhante aos resultados de laboratório. A taxa de lagartas parasitadas não foi diferente entre as diferentes densidades de hospedeiro oferecidas $(25,50,85$ e 100) ( $>0,05)$. Também não houve interação significativa entre o tempo após liberação e a densidade de lagartas nas microparcelas $(\mathrm{p}>0,05)$.

Entre várias características, o aumento na taxa de lagartas parasitadas, como resposta à maior disponibilidade de hospedeiros, sugere que o parasitoide tem potencial de regulação populacional da praga (Godfray, 1994). Algumas espécies de parasitoides aumentam a taxa de parasitismo mediante a densidade do hospedeiro (Faria et al., 2000, 2008; Sampaio et al., 2001; Makundi \& Sariah, 2005). Em O. sokolowskii, a resposta funcional não apresentou incremento na taxa de parasitismo com aumento da densidade do hospedeiro, e essa espécie parece apresentar uma resposta funcional do tipo I, ou seja, a taxa de parasitismo é mantida constante independentemente da densidade de hospedeiros. Resultados semelhantes foram encontrados por Silva-Torres et al. (2009a), que avaliaram um número constante e excedente de lagartas de $P$. xylostella versus um número variável de lagartas diariamente ofertadas a $O$. sokolowskii. O parasitoide também parasitou um número constante de lagartas por dia, abaixo do número máximo oferecido, mesmo quando submetidos a condições prévias de escassez de hospedeiro. Por sua vez, Chen et al. (2008) relataram que, quando densidades de 20 até 50 lagartas de $P$. xylostella foram oferecidas a $O$. sokolowskii, por 48 horas, em laboratório, a taxa de parasitismo diminuiu de maneira gradativa e inversamente proporcional ao aumento da disponibilidade de hospedeiro, indicando assim uma resposta funcional tipo II. Baseado nesses resultados, Chen et al. (2008) sugerem que esse parasitoide não poderia regular a praga em altas densidades. Esses autores também observaram que fêmeas de O. sokolowskii são capazes de causar interferência mútua, provavelmente pela marcação do hospedeiro com feromônio, reduzindo assim o número de lagartas atacadas por cada parasitoide. Esta interferência mútua entre fêmeas de $O$. sokolowskii pode ter ocorrido neste trabalho, uma vez que foram utilizados grupos de fêmeas para avaliação do parasitismo em diferentes densidades do hospedeiro. Portanto, a taxa de parasitismo final obtida tornou-se reduzida.

Outra hipótese que poderia explicar a constante taxa de parasitismo apresentada por $O$. sokolowskii neste trabalho seria a produção e a maturação de ovos ao longo da vida adulta, como ocorre com parasitoides sinovigênicos (Papaj, 2000). Parasitoides sinovigênicos não sofrem interferência da disponibilidade de hospedeiros, uma vez que eles só parasitam um número máximo de ovos por dia, independentemente de uma oferta maior de hospedeiros. No entanto, estudos fisiológicos sobre a produção e maturação de ovos

Tabela 1. Percentagem média de parasitismo de lagartas de Plutella xylostella, em diferentes densidades de infestação, por Oomyzus sokolowskii em casa telada, em função da densidade média de lagartas recuperadas após 24 ou 72 horas de exposição ao parasitismo.

\begin{tabular}{|c|c|c|c|c|}
\hline \multirow[t]{2}{*}{ Densidade inicial $^{(1)}$} & \multicolumn{2}{|c|}{24 horas } & \multicolumn{2}{|c|}{72 horas } \\
\hline & Lagartas recuperadas & Taxa de parasitismo & Lagartas recuperadas & Taxa de parasitismo \\
\hline 25 & $18,2 \quad(9-23)$ & $54,4 \pm 11,97$ & $17,5 \quad(8-25)$ & $82,5 \pm 6,62^{\text {ns }}$ \\
\hline 50 & $36,2(20-50)$ & $57,3 \pm 8,52$ & $32,6(13-47)$ & $81,5 \pm 5,00 *$ \\
\hline 85 & $64,4(32-85)$ & $48,9 \pm 10,63$ & $25,6(16-42)$ & $81,3 \pm 2,73 *$ \\
\hline 100 & $69,6(54-95)$ & $49,0 \pm 12,32$ & $62,2(42-80)$ & $77,5 \pm 5,38^{\mathrm{ns}}$ \\
\hline
\end{tabular}

${ }^{(1)}$ Densidade inicial de lagartas infestadas correspondente a seis plantas de repolho por microparcela. Valores entre parênteses significam os números máximo

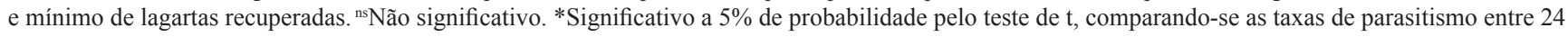
e 72 horas. 
em O. sokolowskii ainda são necessários para maiores esclarecimentos.

A disponibilidade e a distribuição espacial do hospedeiro, entretanto, exercem papel importante no comportamento de parasitismo de O. sokolowskii. Quando os parasitoides foram liberados nos telados, com uma variabilidade na distribuição espacial do hospedeiro, além de oferecer a possibilidade de dispersão, os parasitoides foram capazes de localizar, agregar e parasitar mais lagartas em áreas com maior infestação da praga. Portanto, os resultados deste trabalho sugerem que, em campo, os parasitoides poderiam deslocar-se tanto para áreas com baixa quanto para áreas com alta infestação do hospedeiro. Porém, ainda mais importante, os parasitoides são capazes de parasitar mais lagartas nas áreas com maior infestação da praga.

É provável que a resposta de parasitismo de O. sokolowskii tenha sido resultado da maior concentração de pistas visuais, como a maior quantidade de lesões nas plantas, e químicas, como a liberação de maior concentração de voláteis, que auxiliaram os parasitoides no processo de localização do hospedeiro em densidades mais elevadas. A intensidade de lesões por planta nas microparcelas pode aumentar consideravelmente com o aumento da infestação da praga. Além disso, plantas podem utilizar substâncias químicas (voláteis) para promover a ação efetiva de inimigos naturais de insetos herbívoros (Van den Boom et al., 2004; Schoonhoven et al., 2005). Estudos comportamentais de respostas a estímulos visuais e olfativos são inexistentes com O. sokolowskii. No entanto, uma resposta positiva a esses estímulos foi identificada em outras espécies de parasitoides (Meiners \& Hilker, 2000; Colazza et al., 2004), incluindo Oomyzus gallerucae (Fonscolombe) (Hymenoptera: Eulophidae), em que fêmeas foram atraídas por voláteis liberados por plantas de Ulmus campestris L. (Urticaceae), infestadas com ovos de Xanthogaleruca luteola (Muller) (Coleoptera: Chysomelidae) (Meiners \& Hilker, 1997). Assim, é possível que fêmeas de $O$. sokolowskii tenham sido atraídas por plantas de repolho atacadas por P. xylostella, em baixas e altas densidades, e que essa atração tenha sido intensificada em áreas com maior infestação da praga. Esse comportamento é desejado para o controle biológico aplicado.
Neste trabalho, houve incremento significativo na taxa de lagartas parasitadas, quando expostas por mais tempo ( 72 horas) aos parasitoides em casa telada. Esse resultado indica que o parasitismo por O. sokolowskii também é dependente do fator tempo, uma vez que a taxa de parasitismo praticamente dobrou, de aproximadamente $40 \%$, em 24 horas, para $80 \%$, em 72 horas de exposição. Foerster et al. (2001) também constataram que houve aumento no parasitismo com o tempo de exposição de larvas de Mythimna (Pseudaletia) sequax Franclemont (Lepidoptera: Noctuidae) ao parasitoide Glyptapanteles muesebecki (Blanchard) (Hymenoptera: Braconidae). É provável que, com o aumento do tempo disponível, os parasitoides sejam estimulados pela maturação de ovos a continuar procurando e tenham maiores chances de localizar e parasitar mais hospedeiros em campo. Os parasitoides sinovigênicos necessitam de tempo para a produção de novos ovos durante a vida adulta e, portanto, são limitados pelo tempo para a expressão de todo o seu potencial de parasitismo (Papaj, 2000).

Em condições de confinamento, em campo, em que a variabilidade ambiental foi maior, incluindo precipitação durante o experimento, mesmo quando os parasitoides tiveram um período de 72 horas para localizar e parasitar o hospedeiro, a taxa de parasitismo foi inferior à encontrada em laboratório. Assim, uma hipótese complementar é a de que, além do tempo de exposição, outros fatores tais como a área de forrageamento e as condições ambientais (temperatura, chuva, vento, intensidade de luz) interagem e podem interferir na capacidade de parasitismo dos parasitoides. Resultados semelhantes foram encontrados por Weisser et al. (1997), ao estudar o impacto de condições climáticas na capacidade de forrageamento e parasitismo de Aphidius rosae Haliday (Hymenoptera: Aphidiidae). Os autores concluíram que o potencial de parasitismo de $A$. rosae, em condições de campo, é variável e inferior às condições de laboratório quando o clima não é favorável. Assim, as variações encontradas na capacidade de parasitismo de O. sokolowskii podem ter sido resultado das diferentes condições experimentais adotadas no laboratório, semicampo e campo, mas ficou evidente que o tempo de exposição é um fator importante para incremento na sua taxa de parasitismo.

Em relação à progênie, o número de descendentes produzidos por lagarta parasitada decresceu 
significativamente com o aumento da densidade do hospedeiro em laboratório $(\mathrm{p}<0,01)$ (Figura 3$)$. Essa circunstância sugere que $O$. sokolowskii é capaz de ajustar sua progênie em função da disponibilidade de hospedeiros, depositando mais ovos por lagarta, quando os hospedeiros disponíveis estão em baixa densidade. Possivelmente, as fêmeas de $O$. sokolowskii são capazes de distribuir a progênie de maneira mais uniforme, quando o hospedeiro está em alta densidade, ou pode ocorrer menor número de encontros com hospedeiros já parasitados.

É provável que isso tenha ocorrido nas condições utilizadas no experimento de laboratório, pela diferença na relação do número de fêmeas progenitoras por hospedeiro disponível. Em baixa densidade de lagartas (2), registrou-se uma média de cinco fêmeas do parasitoide por lagarta, enquanto na densidade mais alta (16) essa relação foi reduzida para 0,6 fêmeas por lagarta. Algumas espécies de parasitoides são capazes de ajustar o número e o sexo dos descendentes em função da relação número de fêmeas progenitoras e disponibilidade de hospedeiro (Godfray, 1994; Burton-Chellew et al., 2008), o que também foi registrado em relação a $O$. sokolowskii por Chen et al. (2008).

Contrariamente aos resultados de laboratório, não houve diferença no número de descendentes de O. sokolowskii produzidos por lagarta parasitada

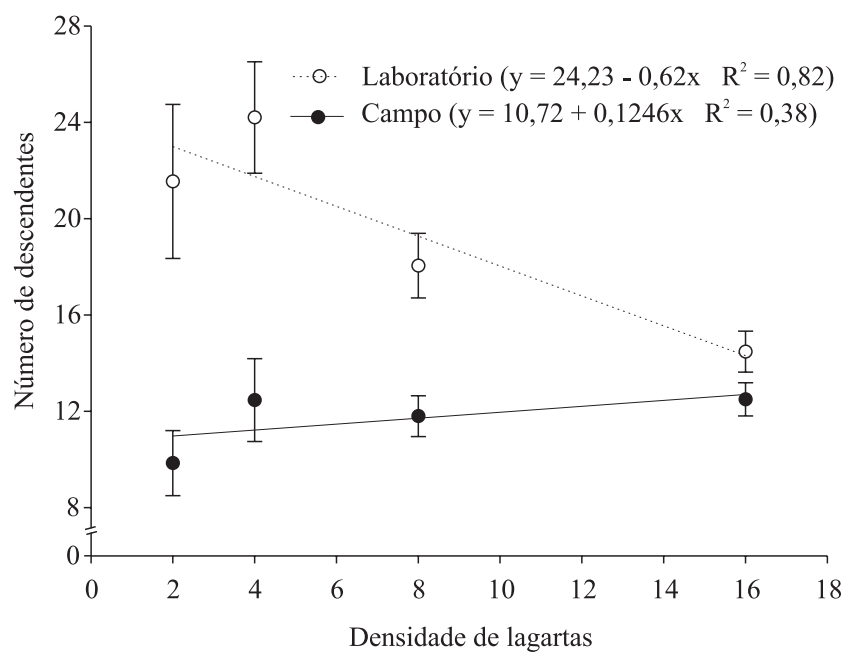

Figura 3. Número médio de descendentes de Oomyzus sokolowskii produzidos por lagarta parasitada de Plutella xylostella durante 48 horas, em laboratório, e 72 horas, em campo, em função de diferentes densidades do hospedeiro. A barra vertical indica o erro-padrão. em condições de microparcelas e campo $(p>0,05)$ (Figura 3). A média de descendentes produzidos por lagartas parasitadas variou de $19,2 \pm 0,99$ a $22,2 \pm 0,83$

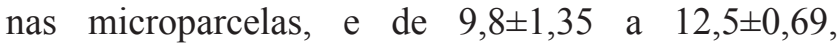
em campo. Possivelmente, essa divergência de resultados ocorreu pelo fato de que, em laboratório, os parasitoides estavam confinados em recipientes de $300 \mathrm{~mL}$, o que possibilitou múltiplos encontros com hospedeiros previamente parasitados, principalmente em densidades baixas, e favoreceu ao superparasitismo (Silva-Torres et al., 2009b). Em condições de microparcelas e campo, os parasitoides tiveram uma área maior para forrageamento, reduzindo assim as chances de encontrarem hospedeiros já parasitados e, consequentemente, de superparasitismo.

\section{Conclusões}

1. Oomyzus sokolowskii tem potencial para localizar e parasitar P. xylostella, seja em altas ou baixas densidades populacionais.

2. O tempo de pelo menos 72 horas após as liberações dos parasitoides é importante para atingir níveis mais elevados de parasitismo.

3. Oomyzus sokolowskii parasita mais lagartas de $P$. xylostella com o aumento da densidade do hospedeiro, entretanto, a taxa de parasitismo é mantida constante.

4. Em condições de casa telada e campo, a produção de descendentes não é afetada por variações na densidade do hospedeiro.

\section{Agradecimentos}

À Coordenação de Aperfeiçoamento de Pessoal de Nível Superior, pela bolsa concedida ao primeiro autor; à Professora Ângela Maria Isidro de Farias, pelas correções e sugestões no manuscrito.

\section{Referências}

BURTON-CHELLEW,M.N.;KOEVOETS, T.; GRILLENBERGER, B.K.; SYKES, E.M.; UNDERWOOD, S.L.; BIJLSMA, K.; GADAU, J.; ZANDE, L. van de; BEUKEBOOM, L.W.; WEST, S.A.; SHUKER, D.M. Facultative sex ratio adjustment in natural populations of wasps: cues of local mate competition and precision of adaptation. American Naturalist, v.172, p.393-404, 2008.

CHEN, R-.X.; ZHANG, F.; HUANGFU, W-.G.; YAO, H-.Y.; ZHOU, J-.B.; KUHLMANN, U. Reproductive attributes of the eulophid Oomyzus sokolowskii, a biological control agent of 
diamondback moth, Plutella xylostella (Lepidoptera: Plutellidae). Biocontrol Science and Technology, v.18, p.753-765, 2008.

COLAZZA, S.; FUCARINO, A.; PERI, E.; SALERNO, G.; CONTI, E.; BIN, F. Insect oviposition induces volatile emission in herbaceous plants that attracts egg parasitoids. Journal of Experimental Biology, v.207, p.47-53, 2004.

FARIA, C.A.; TORRES, J.B.; FARIAS, A.M.I. Resposta funcional de Trichogramma pretiosum Riley (Hymenoptera: Trichogrammatidae) parasitando ovos de Tuta absoluta (Meyrick) (Lepidoptera: Gelechiidae): efeito da idade do hospedeiro. Anais da Sociedade Entomológica do Brasil, v.29, p.85-93, 2000.

FARIA, C.A.; TORRES, J.B.; FERNANDES, A.M.V.; FARIAS, A.M.I. Parasitism of Tuta absoluta in tomato plants by Trichogramma pretiosum Riley in response to host density and plant structures. Ciência Rural, v.38, p.1504-1509, 2008.

FERREIRA, S.W.J.; BARROS, R.; TORRES, J.B. Exigências térmicas e estimativa do número de gerações de Oomyzus sokolowskii (Kurdjumov) (Hymenoptera: Eulophidae), para regiões produtoras de crucíferas em Pernambuco. Neotropical Entomology, v.32, p.407-411, 2003.

FOERSTER, L.A.; DOETZER, A.K.; AVANCI, M.R.F. Parasitoides larvais de Mythimna (Pseudaletia) sequax Franclemont e capacidade de parasitismo de Glyptapanteles muesebecki (Blanchard) em relação ao tempo de exposição, temperatura e densidade de hospedeiros. Acta Biológica Paranaense, v.30, p.139-149, 2001.

GODFRAY, H.C.J. Parasitoids: behavioral and evolutionary ecology. Princeton: Princeton University, 1994. 473p.

HASSELL, M.P. The dynamics of arthropod predator-prey systems. Princeton: Princeton University Press, 1978. 237p.

HOLLING, C.S. Principles of insect predation. Annual Review of Entomology, v.6, p.163-182, 1961.

MAKUNDI, R.H.; SARIAH, J.E. A functional response of braconid parasitoids of the bean stem maggot, Ophiomyia spencerella (Diptera, Agromyzidae), in beans (Phaseolus vulgaris L.) in Tanzania. Journal of Plant Diseases and Protection, v.112, p.478-484, 2005.

MEINERS, T.; HILKER, M. Host location in Oomyzus gallerucae (Hymenoptera: Eulophidae), an egg parasitoid of the elm leaf beetle Xanthogaleruca luteola (Coleoptera: Chrysomelidae). Oecologia, v.112, p.87-83, 1997.

MEINERS, T.; HILKER, M. Induction of plant synomones by oviposition by a phytophagous insect. Journal of Chemical Ecology, v.26, p.221-232, 2000.
PAPAJ, D.R. Ovarian dynamics and host use. Annual Review of Entomology, v.45, p.423-448, 2000.

PRATISSOLI, D.; VIANNA, U.R.; REIS, E.F. dos; ANDRADE, G.S.; SILVA, A.F. da. Influência da densidade de ovos de Spodoptera frugiperda em alguns aspectos biológicos de três espécies de Trichogramma. Revista Brasileira de Milho e Sorgo, v.4, p.1-7, 2005.

SAMPAIO, M.V.; BUENO, V.H.P.; PÉREZ-MALUF, E.R. Parasitismo de Aphidius colemani Viereck (Hymenoptera: Aphidiidae) em diferentes densidades de Myzus persicae (Sulzer) (Hemiptera: Aphididae). Neotropical Entomology, v.30, p.81-87, 2001.

SAS INSTITUTE. SAS/STAT: user`s guide: statistics. Version 8. Cary: SAS Institute, 2000.

SCHENK, D.; BACHER, S. Functional response of a generalist insect predator to one of its prey species in the field. Journal of Animal Ecology, v.71, p.524-531, 2002.

SCHOONHOVEN, L.M.; LOON, J.J.A. van; DICKE, M. Insect-plant biology. New York: Oxford University, 2005, 421P.

SILVA-TORRES, C.S.A.; BARROS, R.; TORRES, J.B. Efeito da idade, fotoperíodo e disponibilidade de hospedeiro no comportamento de parasitismo de Oomyzus sokolowskii Kurdjumov (Hymenoptera: Eulophidae). Neotropical Entomology, v.38, p.512-519, 2009a.

SILVA-TORRES, C.S.A.; RAMOS FILHO, I.T.; TORRES, J.B.; BARROS, R. Superparasitism and host size effects in Oomyzus sokolowskii, a parasitoid of diamondback moth. Entomologia Experimentalis et Applicata, v.133, p.65-73, 2009 b.

VAN DEN BOOM, C.E.M.; VAN BEEK, T.A.; POSTHUMUS, M.A.; DE GROOT, A.E.; DICKE, M. Qualitative and quantitative variation among volatile profiles induced by Tetranychus urticae feeding on plants from various families. Journal of Chemical Ecology, v.30, p.69-89, 2004.

WAAGE, J.K. Aggregation in field parasitoid populations: forage time allocation by a population of Diadegma (Hymenoptera, Ichneumonidae). Ecological Entomology, v.8, p.447-459, 1983.

WANG, X-.G.; LIU, S-.S.; GUO, S-.J.; LIN, W-.C. Effects of host stages and temperature on population parameters of Oomyzus sokolowskii, a larval-pupal parasitoid of Plutella xylostella. BioControl, v.44, p.391-403, 1999.

WEISSER, W.W.; VÖLKL, W.; HASSELL, M.P. The importance of adverse weather conditions for behavior and population ecology of an aphid parasitoid. Journal of Animal Ecology, v.66, p.386-400, 1997.

Recebido em 14 de janeiro de 2010 e aprovado em 28 de junho de 2010 